\title{
EDITORIAL
}

\section{Has the time come to shift to paper DICOM printing?}

\section{Anirudh Kohli}

Department of Imaging, Breach Candy Hospital Trust, Mumbai, Maharashtra, India. E-mail: dranirudhkohli@gmail.com

In the 1990s when we started doing cross-sectional imaging, the scenario was very different than today. There was no picture archiving and communication system (PACS), and there was a single monitor to do planning of scans and viewing of images. Rarely there was luxury of a slave monitor, where images could be seen without coming in the way of the technologist, who were the big bosses then, shooing radiologist away from the console monitor - their domain!

The minimum slice thickness was usually 5/8/10 mm, so all images were documented on film directly. Reporting was done from films. Since all the diagnostic information needed was captured on film. Good quality film cameras, films, and view boxes were as important as good quality scanners. Subsequently, to facilitate the reading process, motorized viewing panels were introduced which cost lakhs of rupees at that time! As the applications were evolving, the regions scanned were limited. Clinicians especially neurologists and neurosurgeons would take pride in their ability to read scans and even do today but to a much lesser extent than those days.

\section{So film was king in that era}

Today, the scenario is totally different with digital X-ray, multislice computed tomography (CT), and parallel imaging magnetic resonance imaging. Imaging data generated are huge. These data are viewed electronically on a monitor either a workstation or PACS. Most PACS have the ability to incorporate various levels of workstation capabilities into their architecture. In fact, nearly all entry-level PACS have all the basic workstation tools. The cost of PACS has tremendously come down. Entry-level PACS cost in the range of 5-10 lakhs and enterprise PACS cost in the range of 40-50 lakhs. Off course prices can be ballooned up by adding numerous licences which are usually not necessary and excessive storage, though storage prices are falling rapidly. There are numerous additional benefits in having data in an electronic format beyond viewing thin slices. Data are now obtained isotropic especially with MDCT; these isotropic data can be acquired in one plane but reconstructed in any plane without any loss of resolution. This is very important in evaluating the extent of lesions, involvement of adjacent structures, eliminating partial volume effects, identifying the organ of origin of lesions, and so on. Other postprocessing techniques are also useful such as window adjustments, maximum intensity projections especially to detect pulmonary nodules and not confuse with end on vessels, minimum intensity projections for detecting airway abnormalities, volume rendering, and both surface contouring and luminal. From a reporting point of view, comparison of previous studies is very important to determine whether there is disease progression or regression. Electronic data set images can be synched to an exact anatomical level and then viewed simultaneously with both data sets moving in sync anatomically. This is a highly accurate and reproducible means of comparison of data sets. The possibilities and advantages of electronic data sets are immense, invaluable, and an absolute necessity today.

CT slices are in the range of $0.6 \mathrm{~mm}$ today. If all images are to be documented, this will result in a large number of films. Data are resliced with a thickness of $5 \mathrm{~mm}$ and documented on film. What is the utility today of these films? Not for the radiologist as reporting is done on viewing thin sections electronically. Films are essentially for the referring clinician and patient. Most clinicians are not so adept at reading films today as they were in the past, as the applications, regions covered, and detail available have tremendously changed. There are numerous questions that arise whether they see the films or not, do they just see the films for the extent of abnormality for themselves, or as a communication tool with patients, to explain the disease process. Very often films are seen only once; a perfect example is in the intensive care unit. Chest $X$-ray films are obtained at least once a day. This is very important to see the state of the lungs especially how wet they are. These $\mathrm{X}$-rays are seen maximum twice, once on the day taken and the next day for comparative purposes and then of no utility. This happens with imaging studies which are normal or have insignificant findings.

A technical issue that happens commonly in imaging departments is that since images are read electronically by the radiologist and the films printed by the technologist, the radiologist rarely views the films. A lazy technologist may result in shoddy documentation of films, thus affecting the 
reputation of the imaging facility and the radiologist may never come to know this!!

One important point missed is the film is expensive, and the price may have floated down for some or stayed static. Today, it is in the range of 70 Rs., plus or minus 10 Rs. based on volume, negotiation capabilities, and so on. A typical cross-sectional study would average at least five films which means approx. 350-400 Rs. per study. X-ray just the consumable cost of film would be $25 \%$ of the examination cost.

Is there an alternative? Yes, at least two broad alternatives - electronic and paper printing. Electronic is a very attractive alternative and is especially possible in institutional setups and in play already at some institutions. Images are available to be seen on any PC or electronic device using a zero foot print viewer. Are these expensive? Not at all. The images could be stored on a server on site or in a cloud storage. The clinician can see images at any location as long as there is Internet to access data. This has further got enhanced with the marked drop in pricing of data packages available. Another electronic option is a CD. Most imaging facilities today provide a CD of the images - though some unfortunately only document the thick slices used for filming; this is a waste and it is important to archive the thinnest of data sets as these will be useful not only for viewing but also post processing at a later date. Since the data are stored in a DICOM format, a DICOM reader is added to the CD. These are freely available and often provided by the PACS vendor. A clinician can also download a free DICOM viewer for Windows or Mac.

The other option is paper printing using DICOM paper printers. The major advantage of paper printing is a drop in cost from 70-80 Rs. for $14 \times 17$ film to a comparable paper size of A3 of 15-20 Rs. There is usually no capital cost of the printer; however, if the user wants to buy the printer in the range of 3-4 lakhs, the cost per paper print will drop. On a single CT study, the saving per study would be in the range of 400 Rs. This is huge more than what most radiologists are paid to read the study!! Hopefully, administrators and owners of centers will pass on some of this saving to radiologists if they transit away from printing films!

What about the acceptability of paper printing? An interesting exercise is PETCT; most PETCT centers print the PET color images on paper and grey scale CT scan images on paper. The oncologist is one clinician category who looks at films to plan surgery or therapy. They have accepted this medium of documentation of images.

Then what is holding the radiologist back from saving money? Most often the answer is clinician acceptance as they are not bothered about cost saving and will insist on conventional films. The other reason is competition - if one imaging center switches to paper and a competing center does not, then maybe the center still providing films will benefit. Though an argument would be the saving on film cost may compensate for the apparent loss of work.

A very important statistic is the film market in India is annually $800-1000$ crores!! All vendors have come up with this figure in personal communications. What does this mean? If radiology in India moved to paper printing from film printing, there would be a saving of $600-750$ crores a year!! And if there is a further shift to electronic, this can be even more!!

To put these figures in perspective, the Bandra-Worli Sea link in Mumbai was built at a total cost of 1500 crores. This would mean if radiologists make a minor shift from film to paper, the saving in 2 years will be equal to building a new Bandra-Worli Sea Link!! The Bandra-Worli Sea Link earns money every day from toll, films don't!!

These figures and comparison seem unreal, but they have been verified and reverified.

It just does not seem logical to continue printing on film, as today it is not used for reporting - the saving can be immense.

This is an open access journal, and articles are distributed under the terms of the Creative Commons Attribution-NonCommercial-ShareAlike 4.0 License, which allows others to remix, tweak, and build upon the work non-commercially, as long as appropriate credit is given and the new creations are licensed under the identical terms.

\begin{tabular}{|c|c|}
\hline \multicolumn{2}{|c|}{ Access this article online } \\
\hline Quick Response Code: & \multirow[b]{2}{*}{$\begin{array}{l}\text { Website: } \\
\text { www.ijri.org }\end{array}$} \\
\hline & \\
\hline & $\begin{array}{l}\text { DOI: } \\
\text { 10.4103/ijri.IJRI_278_19 }\end{array}$ \\
\hline
\end{tabular}

Cite this article as: Kohli $\mathrm{A}$. Has the time come to shift to paper DICOM printing? Indian J Radiol Imaging 2019;29:109-10. 